\title{
Efeitos da Modelação e Reforçamento Positivo no Consumo de Frutas em Crianças
}

\author{
Makerley Stefanine Silva Ferreira* (®, Sônia Maria Mello Neves (®,

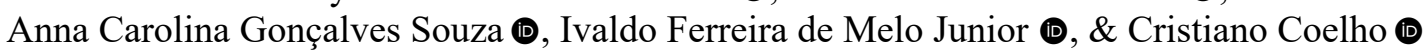 \\ Pontifícia Universidade Católica de Goiás, Goiânia, GO, Brasil
}

\begin{abstract}
RESUMO - Este estudo avaliou o efeito da modelação (vídeos) e das consequências sobre o consumo de frutas em oito pré-escolares. No Experimento1 foram apresentados vídeos e, posteriormente, consequências. No Experimento 2 foram programadas consequências, em seguida, vídeos e consequências conjuntamente. No Experimento 1, o consumo foi maior durante apresentação de consequências; no Experimento 2, a apresentação conjunta de vídeos e consequências produziu maior consumo que consequências isoladamente, sugerindo efeito potencializador dos vídeos. Mudança gradual na intermitência das consequências reduziu consumo de três participantes do Experimento 1, mas aumentou consumo para os dois participantes afetados pelas intervenções no Experimento 2. O consumo foi maior na LBII comparada à LBI, porém menor que durante intervenções, indicando efeito temporário dessas intervenções sobre o consumo.
\end{abstract}

PALAVRAS-CHAVE: hábitos alimentares, frutas, modelação, reforço positivo, Análise do Comportamento

\section{Effects of Modeling and Positive Reinforcement on Children's Fruit Consumption}

\begin{abstract}
This study evaluated the effects of modeling (videos) and consequences on fruit consumption of three preschool children. In Experiment 1, videos were presented, and subsequently, consequences. In Experiment 2, consequences were programmed, then videos and consequences were presented together. In Experiment 1, consumption was greater during presentation of consequences; in Experiment 2, videos and consequences together produced higher consumption than only consequences, suggesting an enhancing effect of videos. Gradual change in the intermittence of consequences reduced consumption for three participants in Experiment 1, but increased consumption for the two participants affected by the interventions in Experiment 2. Consumption was greater in BLII, compared to BLI, but it was lower than during interventions, indicating temporary effect of such interventions on consumption.
\end{abstract}

KEYWORDS: eating habits, fruit, modeling, positive reinforcement, Behavior Analysis

A alta incidência de doenças crônicas não transmissíveis (DCNT) como, por exemplo, hipertensão, diabetes e obesidade, respondem por mais de $70 \%$ das causas de mortes no Brasil atualmente (Brasil, 2014). Segundo a Organização Mundial da Saúde (WHO, 2014) e a Organização das Nações Unidas para a Alimentação e a Agricultura (FAO, 2007), as DCNT convivem com alta prevalência de deficiências nutricionais, sendo o excesso de peso, os níveis elevados de colesterol e o baixo consumo de frutas, legumes e verduras (FLV) considerados fatores de risco importantes associados à elevada morbidade e mortalidade.
O consumo mínimo de FLV recomendado pela WHO (2003) é de 400 gramas por dia ou o equivalente a cinco porções para adultos e crianças (a porção desses alimentos varia entre 80 e 150 gramas). No Brasil, o Ministério da Saúde (2005), em seu Guia Alimentar para a População Brasileira, recomenda o consumo diário mínimo de três porções de frutas e três porções de legumes e verduras para pessoas saudáveis com idade igual ou superior a dois anos.

O Ministério da Saúde (2014) avaliou o consumo de FLVs pela população brasileira a partir do sistema Vigitel (Vigilância de Fatores de Risco e Proteção para Doenças

\footnotetext{
*E-mail: makerleystefanini@gmail.com

- Submetido: 10/02/2019; Aceito: 19/09/2019.
} 
Crônicas por Inquérito Telefônico). Essa consulta mostrou que apenas $25 \%$ dos brasileiros consomem a quantidade de FLV recomendada pela WHO (2003), e a ingestão geral da população é de apenas $33 \%$ do recomendado. Ao avaliar o consumo de frutas em crianças, pesquisadores constataram que, na faixa etária de dois a cinco anos, apenas 25,2\% consumiam frutas entre cinco e sete vezes por semana, e na faixa de cinco a 10 anos, apenas $38 \%$ informaram ingerir frutas diariamente (Ministério da Saúde, 2009).

Crianças e adolescentes são alvos preferenciais para intervenções que incentivam o consumo de FLV, já que são mais propensas a mudanças comportamentais do que adultos (Singer et al., 1995; Stice, 1998). Além disso, suas preferências alimentares permanecem relativamente estáveis e se refletem nas escolhas de alimentos feitas na vida adulta (Damiani et al., 2017; Mikkilä et al., 2004).

Em um estudo realizado por Costa (2012), foi avaliada a ingestão de FLV entre 4.964 crianças brasileiras de seis a 10 anos, de ambos os sexos. O consumo considerado adequado, seguindo critérios da WHO (2003), de cinco ou mais porções diárias, esteve presente em $2,7 \%$ dos escolares, enquanto $26,6 \%$ não consumiram frutas e hortaliças sequer uma vez por dia. O estudo de Bigio et al. (2010), realizado com 812 adolescentes brasileiros de ambos os sexos, objetivou identificar fatores associados ao consumo de FLV. Os autores identificaram que apenas $6,4 \%$ dos adolescentes consumiam a recomendação mínima de cinco ou mais porções, $22 \%$ não consumiam nenhum tipo de FLV, e que a renda domiciliar per capita e a escolaridade do chefe de família associavamse positivamente ao consumo. Em conjunto, esses resultados revelam baixos percentuais de consumo regular de FLV por crianças e adolescentes brasileiros, apontando para a necessidade de ações urgentes com essas populações, objetivando favorecer mudanças de hábitos alimentares.

Segundo Horne et al. (2004), poucos trabalhos têm sido desenvolvidos para garantir que o consumo de FLV seja suficiente (ver também Pereira et al., 2017). Além disso, alguns estudos não demonstraram, de forma sistemática, a influência dos métodos ou intervenções adotados, tais como o fornecimento de orientações nutricionais e informações sobre os riscos de não se consumir alimentos saudáveis (Baranowski, 2000; Damiani et al., 2017; Domel et al., 1993).

Estudos desenvolvidos ao longo de 25 anos na Universidade Bangor, sob a liderança de Pauline Horne e Fergus Lowe, com base nos princípios e intervenções da Análise do Comportamento e dos achados da Psicologia do Desenvolvimento, deram origem ao Food Dudes Program (Super-Heróis da Alimentação, em tradução livre). Esse programa foi lançado em todas as escolas primárias da Irlanda, tendo recebido os prêmios World Health Organization Counteracting Obesity Award, pela Organização Mundial de Saúde (OMS), em 2006, e Gold Medal at the Chief Medical Officer's Public Health Awards pelo Department of Health and Social Care - UK, em 2010 (Erjavec et al., 2012).

De acordo com os estudos de Horne, Lowe e colaboradores, a exposição ao sabor dos alimentos, a programação de consequências reforçadoras para o comportamento alimentar e a presença de modelos alimentares adequados seriam os principais fatores determinantes das escolhas alimentares de crianças (Horne et al., 1998). Dessa forma, o Programa Food Dudes possui duas fases principais. Na Fase 1, as crianças assistem, diariamente, a episódios de um DVD especialmente desenvolvido para o programa, ou leem uma carta enviada pelos food dudes (heróis que fornecem modelos de consumo de alimentos saudáveis com o objetivo de que seus comportamentos sejam imitados pelas crianças). Posteriormente, são oferecidas para as crianças frutas e vegetais e aquelas que comem esses alimentos recebem brindes (e.g., brinquedos), visando encorajar as crianças a repetir a degustação desses alimentos. Na Fase 2, o programa continua a apoiar o consumo. Nessa fase são usados gráficos e tabelas de registro de consumo nas salas de aula $\mathrm{e}$ as crianças ganham prêmios e certificados à medida que consomem esses alimentos. O uso desse programa tem gerado aumentos no consumo de FLV, principalmente entre crianças que não comiam ou apresentavam consumo baixo desses alimentos (Horne et al., 2004; Lowe et al., 2004).

A exposição gustativa (provar os alimentos) é amplamente aceita como um determinante importante para adquirir preferências alimentares (Birch, 1987; Birch \& Marlin, 1982; Cooke, 2007; Lakkakula et al., 2011; Skinner, 1980; Wardle et al., 2003). As crianças são mais propensas a comer açúcares e gorduras por seu sabor do que por qualquer consequência deliberadamente programadas por outros. No entanto, para o aumento e manutenção do consumo de FLVs é necessária não somente a exposição gustativa a esses alimentos, mas também a programação de contingências reforçadoras para o consumo dos mesmos (Horne et al., 2011).

Diz-se que o comportamento de comer um determinado alimento foi reforçado quando suas consequências aumentam a probabilidade futura de ocorrência desse comportamento (Skinner, 1980). Pesquisas têm mostrado que as consequências de um comportamento são mais eficazes (i.e., são reforçadores mais potentes) quando são altamente desejáveis e quando o comportamento é visto pela criança como importante (Cameron et al., 2001; Deci et al., 1999; Dickinson, 1989; Prevedini \& Carnevali, 2016), um resultado que tem sido verificado também no âmbito do comportamento alimentar (Grubliauskiene et al., 2012; Lowe, Dowey, \& Horne, 1998). A mídia e grandes empresas de fast food e de produtos industrializados utilizam, amplamente, a oferta de brindes como consequência do consumo de alimentos, em particular, de alimentos não saudáveis (Moura, 2010).

A modelação, i.e., a aprendizagem de novos comportamentos por meio da observação de comportamentos de 
outros indivíduos (Catania, 1998), por sua vez, tem se mostrado particularmente eficaz quando o comportamento do modelo gera consequências reforçadoras (ou punitivas), o modelo e o observador têm idades iguais ou o modelo é um pouco mais velho, e quando o modelo é apreciado ou admirado pelo observador (Horne et al., 2004). Horne et al. (2004), Horne et al. (1998) e Horne et al. (1995) utilizaram o Programa Food Dudes para investigar a eficácia de uma intervenção baseada em modelação por meio de vídeos e reforçamento positivo do comportamento de comer FLV em crianças de dois a 11 anos. Os resultados indicaram que as práticas alimentares de crianças foram maleáveis e que os aumentos de consumo de FLV foram significativos não somente durante as intervenções, mas também no decorrer dos follow-ups com duração média de quatro meses. Os princípios de modelação também têm sido utilizados pela publicidade e mídia de produtos alimentícios (Halpern, 2003). Um estudo realizado por Borzekowski e Robinson (2001) sugeriu que somente uma ou duas exposições a um comercial na TV, com duração entre 10 a $30 \mathrm{~s}$, pode influenciar a preferência por determinados produtos em crianças de dois a seis anos. Adicionalmente, Ueda et al. (2014) enfatizam que a publicidade reconhece o papel não somente dos pais, mas também de amigos, personagens de desenhos, artistas e mascotes nas escolhas alimentares realizadas por crianças.

Em suma, estudos empíricos (e.g., Horne et al., 2009, Horne et al., 2011) sobre a aplicação do Programa Food Dudes têm demostrado que a degustação alimentar, o reforçamento positivo e a modelação têm promovido o consumo de FLVs em crianças de países como Itália, Irlanda e Estados Unidos. Considerando a efetividade desse programa e o baixo consumo de FLVs por crianças brasileiras, seria relevante verificar experimentalmente os efeitos desses procedimentos com crianças brasileiras. Sendo assim, a proposta do presente estudo foi comparar os efeitos: (1) da modelação com vídeos públicos (i.e., desenhos animados) que apresentam personagens populares no Brasil consumindo frutas e incentivando seu consumo; e (2) do uso de reforçadores tangíveis (i.e., adesivos e brinquedos) e sociais (i.e., elogios) sobre o consumo de frutas em crianças pré-escolares brasileiras. A eficácia desses procedimentos foi investigada quando apresentados isoladamente ou em conjunto. Finalmente, em ambos os experimentos foi avaliado o efeito da mudança gradual na intermitência da apresentação das consequências programadas sobre o consumo de frutas.

\section{MÉTODO}

No presente estudo, foram realizados dois experimentos em que duas variáveis independentes (VI) foram manipuladas: a apresentação de vídeos e a liberação de consequências - tangíveis (adesivos e brinquedos) e sociais (elogios) - para o comportamento de consumir frutas. A variável dependente (VD) foi o consumo de frutas (laranja, maçã, melancia, banana e mamão), oferecidas em porções de 120 a $150 \mathrm{~g}$ e cortadas em cubos de aproximadamente $5 \mathrm{~cm}$. O Experimento 1 avaliou o efeito da apresentação isolada de vídeos e de reforços positivos, enquanto o Experimento 2 avaliou os efeitos da apresentação conjunta desses dois procedimentos.

A permissão para realizar o estudo foi concedida pelo Comitê de Ética da Pontifícia Universidade Católica de Goiás (Código - 34232814.2.0000).

\section{Participantes e Local}

Participaram do Experimento 1 quatro crianças (duas do sexo feminino e duas do sexo masculino), com idade entre três e quatro anos, de um Centro Municipal de Educação Infantil (CMEI) para filhos de servidores públicos do Centro Administrativo de Goiânia - Goiás. Participaram do Experimento 2 quatro crianças, com idade entre dois e três anos, todas do sexo masculino, de uma escola privada de Educação Infantil em Goiânia. Ambas as escolas ficam na mesma região da cidade e a faixa econômica das famílias era similar.

Os critérios utilizados para seleção dos participantes foram: (1) as primeiras crianças, resistentes ao consumo de frutas, a serem indicadas por consenso entre diretora, professoras e auxiliares, e (2) autorização dos pais ou responsáveis por cada participante a partir do preenchimento do Termo de Consentimento Livre e Esclarecido (TCLE) e do Termo de Restrição Alimentar (TRA). O TRA consistiu na descrição dos alimentos que seriam usados e na verificação da existência ou não de restrições ao consumo de tais alimentos pelas crianças.

Devido aos critérios de seleção, não foi possível alcançar o número necessário de participantes para os dois experimentos na primeira escola (pública), sendo, então, realizada a seleção em outra escola (privada).

\section{Materiais e Equipamentos}

Nos Experimentos 1 e 2 foram utilizados: crachás de identificação para cada participante; Fichas de Registro do Consumo de Frutas, nas quais os observadores registravam o consumo; e consequências tangíveis para o consumo de alimento, i.e., adesivos e brinquedos (e.g., pulseiras, apitos, carrinhos, relógios). Nas sessões de mídia, foram apresentados cinco vídeos infantis com personagens populares que incentivavam o consumo de frutas (Turma da 
Mônica, Disney, Doki - Episódios 1 e 2, e Salada de Frutas), com duração de 1 a 1,5 min.

Para exibição dos vídeos, no Experimento 1 foi utilizada uma televisão de 32 polegadas conectada a um notebook com tela de 14 polegadas e, no Experimento 2, um projetor data show conectado ao mesmo notebook. Em ambos os experimentos foi utilizada uma câmera para filmagem das sessões experimentais.

O Experimento 1 ocorreu em uma sala de audiovisual da escola, medindo 4,0 × 3,5 m. Os participantes entraram juntos na sala e foram conduzidos até um banco acolchoado de quatro lugares, situado em frente à uma mesa medindo $150 \times 48 \times 60 \mathrm{~cm}$. Sobre a mesa havia cinco vasilhas redondas de plástico transparente, com $17 \mathrm{~cm}$ de diâmetro e $10 \mathrm{~cm}$ de altura. Cada vasilha continha 30 porções de uma fruta específica. Do lado de fora de cada vasilha havia um cartão com a figura da fruta inteira. Neste experimento cada participante dividia as 30 porções de frutas de cada vasilha com os outros três participantes, o que significa em média 7,5 porções de cada frutas por participante e um total médio de 37,5 porções de frutas por sessão por cada participante.

O Experimento 2 foi conduzido em uma sala de audiovisual da escola, com medida de 3,5 x 4,0 m. Os participantes entravam na sala juntos e se sentavam em carteiras individuais com assento, encosto e braço de fórmica, dispostas em formato de círculo. Foi colocada no braço da carteira de cada criança uma bandeja redonda, de plástico, com $18 \mathrm{~cm}$ de diâmetro e $8,5 \mathrm{~cm}$ de altura. Cada bandeja continha cinco potes plásticos de $8,5 \mathrm{~cm}$ de diâmetro e $7 \mathrm{~cm}$ de altura. Em cada pote foram colocadas oito porções de um tipo de fruta e na parte externa de cada pote havia a figura da fruta inteira. Cada participante recebia sua própria bandeja com os cinco potes com frutas, com total de 40 porções de frutas disponíveis por sessão, em todas as fases desse experimento.

Em ambos experimentos, se as crianças comessem todas as porções disponíveis de frutas havia a possibilidade de serem adicionadas mais porções nas vasilhas, mas isso nunca ocorreu.

\section{Procedimento}

As sessões ocorreram diariamente, em dias letivos e, em todas, os participantes eram conduzidos juntos à sala de coleta de dados pelo experimentador. As crianças recebiam o crachá de identificação, sentavam-se e recebiam instruções de acordo com a fase. Em todas as sessões de ambos os experimentos, após o fornecimento das instruções e/ou a apresentação dos vídeos, os participantes tinham 7 min para consumir as frutas (esse foi o tempo médio de consumo observado durante a linha de base), sendo a sessão finalizada após esse período. Em todas as fases, o consumo de fruta de cada participante foi registrado por três observadores independentes.
No Experimento 1, as fases foram programadas conforme descrição a seguir.

\section{Fase I - Linha de Base I (LBI)}

No início de cada uma das cinco sessões dessa fase, os participantes receberam a seguinte instrução: "Vocês podem comer o que quiserem, o quanto quiserem". Nenhum vídeo foi mostrado e nenhuma consequência foi programada.

\section{Fase 2 - Modelação (Apenas vídeos)}

Essa fase compreendeu cinco sessões, sendo cada sessão iniciada com a apresentação de um dos cinco vídeos selecionados, conforme a seguinte sequência: Turma da Mônica, Disney, Doki - Episódios 1 e 2, e Salada de Frutas. Após a exibição do vídeo, as frutas eram servidas e era apresentada a seguinte instrução: Vocês podem comer o que quiserem, o quanto quiserem. Nenhuma consequência foi programada para o consumo de frutas.

\section{Fase 3 - Consequências}

Nessa fase foram realizadas sete sessões. No início de cada sessão, os participantes recebiam a seguinte instrução: Vocês podem comer o que quiserem, o quanto quiserem. A cada pedaço de fruta que comerem, vocês poderão escolher um brinquedo. Se comerem três tipos diferentes de fruta, poderão escolher também um adesivo no final. Ao comer um pedaço de fruta, a criança recebia a consequência verbal (e.g., Muito bem!, É isso ai!!, Parabéns por comer frutas! etc.) e também consequências tangíveis (brinquedos, no caso do consumo de uma porção de fruta, e adesivos, quando três frutas diferentes eram consumidas) que podiam ser levadas para casa. Assim, a liberação da consequência verbal e dos brinquedos foi programada de acordo com um esquema de reforçamento contínuo (CRF).

\section{Fase 4 - Mudança Gradual}

Nessa fase foram realizadas seis sessões, durante as quais foi programado o aumento gradual na intermitência entre reforços (i.e., elogios e brinquedos) com o objetivo de promover variabilidade na escolha das frutas, e assim, ocasionar a experiência com sabores diferentes. Os vídeos não eram apresentados. A cada duas sessões, as consequências verbais e uma das consequências tangíveis (i.e., o brinquedo) eram liberadas de acordo com um esquema de reforçamento de razão fixa, sendo o valor da razão aumentado gradativamente (FR 3, FR 5 e FR 7). Os adesivos foram liberados da mesma forma que nas fases anteriores. Em todas as sessões dessa fase foi oferecida a seguinte instrução: Vocês podem comer o que quiserem, o quanto quiserem. A cada [três ou cinco ou sete, de acordo com a etapa] porções de fruta que comerem, vocês poderão escolher um brinquedo. Se comerem três tipos diferentes de frutas, poderão escolher também um adesivo no final. 
Fase 5 - Linha de Base II (LBII)

Nessa fase, os procedimentos das cinco sessões foram idênticos aos da Fase 1 - LBI.

As fases do Experimento 2 são descritas a seguir.

Fase I - Linha de Base I (LBI)

Nessa fase foram realizadas quatro sessões com procedimentos idênticos aos da Fase 1- LBI do Experimento 1.

\section{Fase 2 - Consequências}

Nessa fase foram realizadas cinco sessões com procedimentos idênticos aos da Fase 3 - Consequências do Experimento 1.

\section{Fase 3 - Modelação e Consequências}

Essa fase compreendeu cinco sessões. Cada sessão era iniciada com a exibição de um dos cinco vídeos selecionados, na mesma ordem em que foram apresentados no experimento anterior. Em seguida, era apresentada a seguinte instrução:
Vocês podem comer o que quiserem, o quanto quiserem. A cada pedaço de fruta que comerem vocês poderão escolher um brinquedo. Se comerem três tipos diferentes de fruta, poderão escolher também um adesivo no final. $\mathrm{O}$ consumo de frutas era seguido por consequências verbais e tangíveis, conforme descrito na Fase 3 - Consequências do Experimento 1.

\section{Fase 4 - Mudança Gradual}

Ocorreram seis sessões nessa fase, as quais incluíam os mesmos procedimentos utilizados na Fase 4 - Mudança Gradual do Experimento 1. Nessa fase ocorreram duas mudanças: o aumento gradual da intermitência dos reforços e a retirada total dos vídeos.

\section{Fase 5 - Linha de Base II (LBII)}

Nessa fase foram realizadas cinco sessões com procedimentos idênticos aos da Fase 1 - LBI do Experimento 1.

$\mathrm{O}$ número de sessões diferiu entre fases e entre experimentos em função do desempenho das crianças.

\section{RESULTADOS}

As Figuras 1 e 2 apresentam o número de porções de frutas consumidas em cada sessão e o consumo médio em cada fase, para cada participante. As médias foram obtidas dividindo-se o número porções de frutas consumidas em cada fase pelo número de sessões realizadas na mesma fase.

\section{Experimento I}

No Experimento 1, na Fase 1 - LBI, o participante AC apresentou consumo médio de 0,8 porção e o participante $\mathrm{AV}$ apresentou o consumo médio de 0,2 porção. Os participantes RA e RE não apresentaram consumo nessa fase. Na primeira intervenção, Fase 2 - Modelação, em que foram apresentados os vídeos, somente o participante $\mathrm{AV}$ mostrou aumento no consumo de frutas, tendo apresentado consumo médio de 1,6 porção. Os participantes RA e RE, similarmente ao que ocorreu na LBI, não consumiram frutas, e o participante AC, apesar de ter consumido frutas na Fase 1 - LBI, também não apresentou consumo nessa fase.

A Fase 3 - Consequências apresentou os maiores consumos de frutas. O participante $\mathrm{AC}$ apresentou consumo médio de 9,3 porções de frutas nessa fase, o participante $\mathrm{AV}$, de 9,0 porções de frutas, e os participantes RA e RE, que não haviam apresentado nenhum consumo nas fases anteriores, consumiram, em média, 8,6 e 6,7 porções de frutas nessa fase, respectivamente. Pode-se observar, na Fase 4 - Mudança Gradual, que o consumo diminuiu para todos os participantes: em média, o participante AC consumiu 1,0 porção, o participante $\mathrm{AV}, 3,3$ porções, o participante RA, 4,7 porções, e o participante RE, 7,8 porções.

Na Fase 5 - LBII, houve uma redução adicional no consumo de frutas de todos os participantes. O participante $\mathrm{AC}$ foi o único que não apresentou nenhum consumo. Os participantes $\mathrm{AV}$, RA e RE apresentaram consumo médio de 1,$2 ; 1,0$ e 1,8 porção, respectivamente.

\section{Experimento 2}

No Experimento 2, o participante MA não consumiu nenhuma porção de frutas no decorrer das fases experimentais e o participante MI consumiu frutas apenas na Fase 2 - Consequências (em média, 0,2 porção). Os demais participantes consumiram frutas em quatro das cinco fases (participante CA) ou em todas as fases (participante JM).

Na Fase 1 - LBI, o participante CA não consumiu frutas, enquanto o participante JM apresentou consumo médio de 0,3 porção de frutas. Na Fase 2 - Consequências, assim como ocorreu no Experimento 1, a apresentação de brinquedos como consequência do comportamento alimentar produziu aumento no consumo de ambos participantes: o participante CA apresentou consumo médio de 1,2 porção e o participante JM, de 6,0 porções de frutas.

$\mathrm{Na}$ Fase 3 - Modelação e Consequências, a exposição aos vídeos e às consequências do consumo geraram aumentos adicionais no consumo dos participantes CA e JM. O consumo do participante CA aumentou para 4,8 porções e o consumo do participante JM aumentou para 9,4 

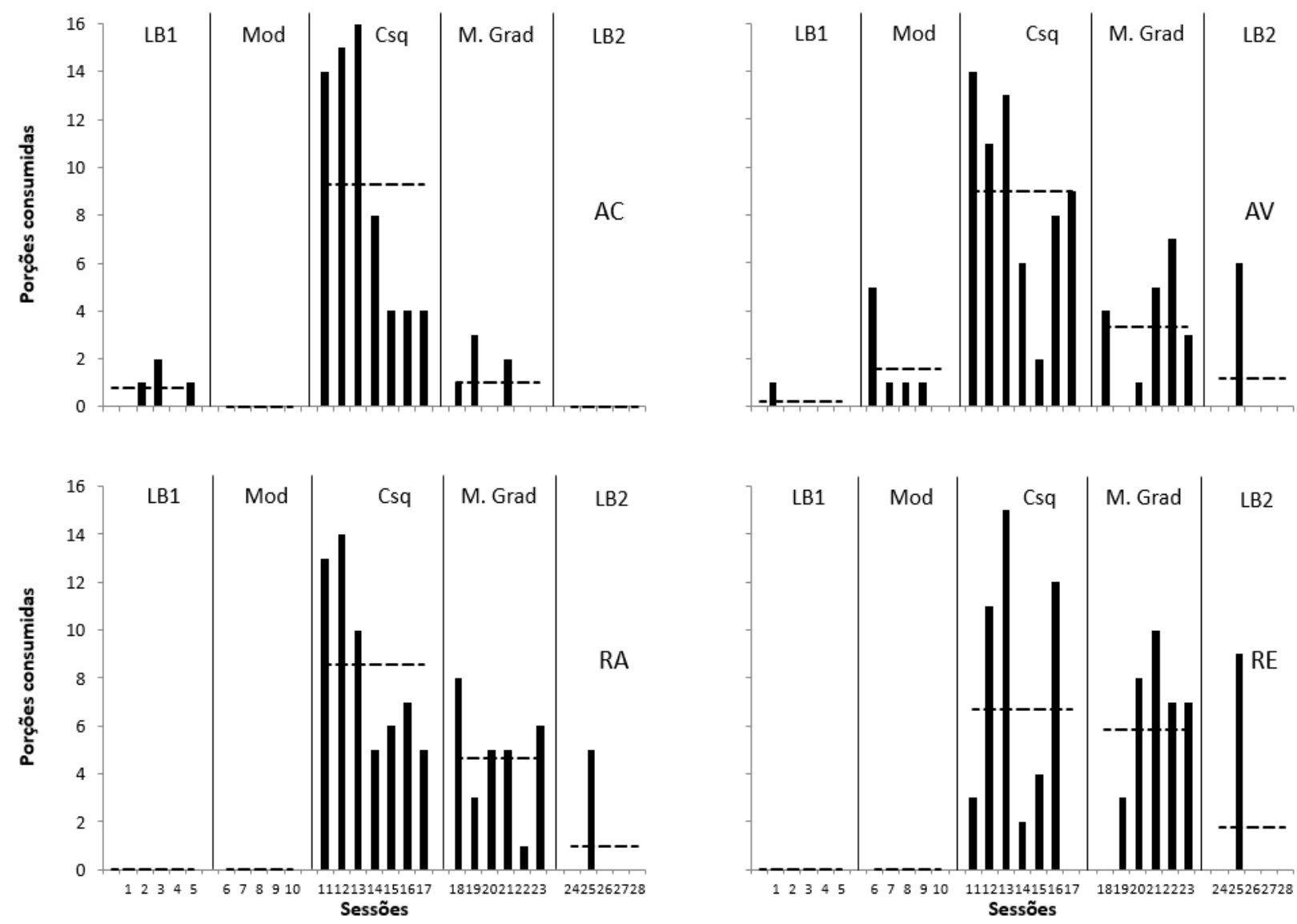

Figura 1. Quantidade de porções consumidas nas fases de Linha de Base 1 (LB1), Modelação (Mod), Consequências (Csq), Mudança Gradual (M. Grad) e Linha de Base 2 (LB2) do Experimento 1 para os participantes AC, AV, RA e RE. As linhas tracejadas referem-se ao consumo médio de cada fase.

porções, em média. Na Fase 4 - Mudança Gradual houve um leve aumento no consumo de frutas, de modo que os participantes CA e JM apresentaram consumo médio de 5,5 e 10,2 porções, respectivamente.

Na Fase 5 - LBII houve uma diminuição no consumo de frutas: o participante CA consumiu, em média, apenas 1,0 porção, enquanto o participante JM teve um consumo médio de 0,6 porção.
Para a análise da precisão do registro dos comportamentos, foi realizado o cálculo do Índice de Concordância entre Observadores (Cooper et al., 2007; Johnston \& Pennypacker, 1993; Kazdin, 1977). O cálculo foi realizado durante pelo menos um terço de todas as sessões de observação, em todas as fases dos experimentos, e para todos os participantes. $\mathrm{O}$ Índice de Concordância do Experimento 1 foi 96,9\% e do Experimento 2 foi $97,9 \%$.

\section{DISCUSSÃO}

O presente estudo avaliou o efeito da modelação por meio do uso de vídeos e de consequências reforçadoras sobre o consumo de frutas de oito crianças pré-escolares. No Experimento 1 foi investigado o efeito da exibição de vídeos e, posteriormente, o efeito das consequências com reforço contínuo e com aumento gradual na intermitência do reforço. Para todos os participantes, o maior consumo de frutas ocorreu quando esse comportamento gerava consequências reforçadoras, principalmente quando o reforçamento era contínuo. No Experimento 2, avaliou-se, inicialmente, o efeito da liberação de consequências contingente ao consumo de frutas e, em seguida, o efeito da combinação da liberação de consequências e do uso de vídeos. Embora a programação de consequências tenha produzido aumentos no consumo de frutas, a apresentação combinada de vídeos e consequências gerou aumentos adicionais nesse consumo. A mudança gradual na intermitência dos reforços produziu efeitos opostos nos dois experimentos, ou seja, diminuiu o consumo no Experimento 1 e aumentou o consumo no Experimento 2. Para cinco dos seis participantes afetados pelas manipulações efetuadas nos dois experimentos, observou-se que o consumo na Fase 5 - LBII foi maior do que na Fase 1 - LBI. 

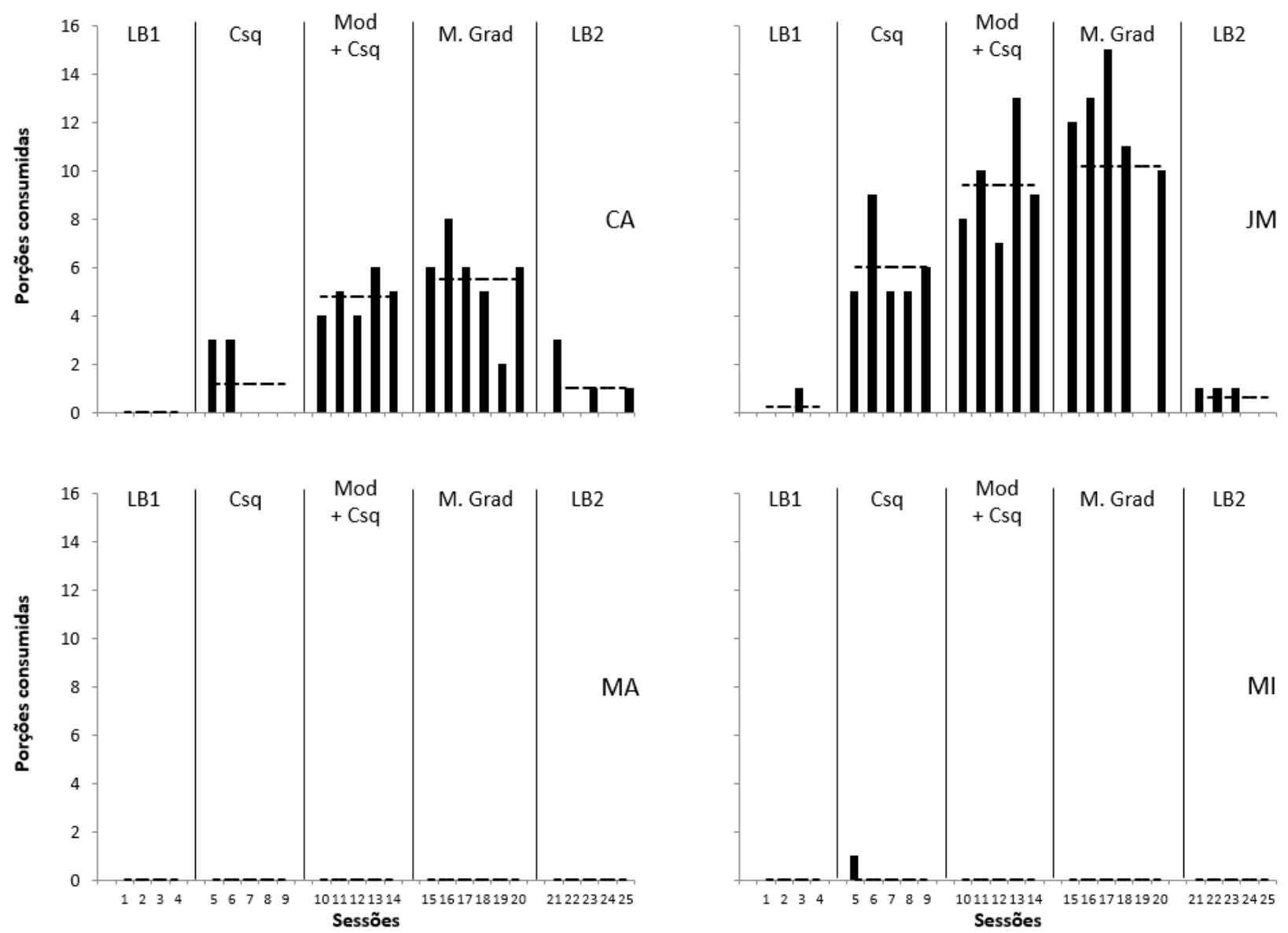

Figura 2. Quantidade de porções consumidas nas fases de Linha de Base 1 (LB1), Consequências (Csq), Modelação + Consequências (Mod + Csq), Mudança Gradual (M. Grad) e Linha de Base 2 (LB2) do Experimento 2 para os participantes CA, JM, MA e MI. As linhas tracejadas referem-se ao consumo médio de cada fase.

Na Fase 1 - LBI do Experimento 1, somente dois participantes consumiram frutas ( $\mathrm{AC}$ e $\mathrm{AV})$, enquanto no Experimento 2 apenas um participante as consumiu (JM), o que indica que a exposição às frutas não garante seu consumo. Esses resultados são consistentes com aqueles encontrados por Horne e Lowe (1998), os quais observaram que exposições repetidas aos alimentos de interesse não tiveram efeito sobre o consumo dos mesmos.

O primeiro objetivo do presente estudo foi avaliar os efeitos da modelação, por meio da apresentação de vídeos (Experimento 1). Foi verificado que a exposição aos vídeos não afetou o consumo de frutas para a maioria dos participantes, um resultado que também corrobora aqueles obtidos por Horne e Lowe (1998). Esses autores verificaram que a modelação por meio de vídeo, sem consequências programadas para o comportamento imitativo, parece ter pouco ou nenhum impacto sobre o consumo de alimentos previamente rejeitados. Adicionalmente, os resultados aqui obtidos, em conjunto com os de Horne e Lowe, mostram que nem sempre a exposição a modelos promove o consumo, conforme sugerido por Borzekowski e Robinson (2001).
No estudo desses autores, uma ou duas exposições a um comercial, com duração entre 10 e $30 \mathrm{~s}$, influenciou a preferência de crianças de 2 a 6 anos por determinados produtos.

O segundo objetivo deste estudo consistiu em verificar o efeito da apresentação de consequências contingentes ao consumo. No Experimento 1, a programação de consequências produziu aumento no consumo de frutas para todos os quatro participantes, sendo esse aumento substancialmente maior do que aquele produzido pela exposição aos vídeos. Quando a apresentação de consequências para o consumo de frutas foi a primeira intervenção utilizada (Fase 2, Experimento 2), também ocorreu um aumento no consumo, mas esse aumento foi menor do que aquele observado na mesma condição durante o Experimento 1. É possível que o aumento substancial no consumo, durante o Experimento 1, tenha sido ocasionado, em parte, pela experiência anterior com os vídeos. Horne e Lowe (1998) também avaliaram o papel do uso isolado de consequências reforçadoras, tendo observado que essa intervenção aumentou o consumo, mas a magnitude desse efeito foi pequena. 
O terceiro objetivo - verificar os efeitos da apresentação conjunta de vídeo e consequências - foi avaliado no Experimento 2. O uso combinado dessas duas intervenções foi acompanhado por um maior consumo de frutas que a apresentação isolada de vídeos (Experimento 1) e de consequências (Experimento 2), corroborando os resultados relatados por Horne e Lowe (1998; ver também Horne et al., 1995; Horne et al., 1998). Apesar de haver suporte na literatura para os resultados aqui obtidos quando vídeos e consequências combinados são comparados com a apresentação anterior de apenas consequências, não é possível descartar a possibilidade de que a experiência com a situação experimental tenha contribuído para o acréscimo no consumo. Para eliminar essa possibilidade, seria necessário que a combinação de vídeos e consequências fosse a primeira intervenção.

O último objetivo do presente estudo se referiu aos efeitos da mudança gradual na intermitência do reforço. No Experimento 1, os participantes não mantiveram o consumo produzido pelo uso isolado de consequências quando a intermitência dessas consequências foi gradualmente aumentada. No Experimento 2, por outro lado, o consumo gerado pelo uso conjunto de modelação e consequências aumentou durante a mudança gradual na intermitência dos reforços. No entanto, para os participantes dos dois experimentos que foram afetados pelas intervenções implementadas, não houve aumento nos tipos de frutas consumidas (com exceção do participante RE), sugerindo um forte controle relacionado à preferência gustativa e à ineficácia do procedimento utilizado (i.e., manipulação na intermitência do reforço) para promover variabilidade nas frutas consumidas.

Dois participantes não consumiram frutas (CA) ou consumiram somente 1 porção (MI) no decorrer do Experimento 2. Pelo menos dois fatores podem ter ocasionado a insensibilidade do consumo desses participantes às contingências programadas. Primeiro, esses participantes eram 1 a 2 anos mais novos que os demais, o que implica diferenças no desenvolvimento do repertório comportamental (Rosales-Ruiz \& Baer, 1997). Segundo, é possível que os brinquedos usados como consequências não tenham funcionado como reforçadores para esses participantes. Sugere-se que, em estudos futuros, a seleção de reforços seja baseada em uma avaliação da preferência entre potenciais estímulos reforçadores (Barrett, 1962).

Alguns aspectos do procedimento podem ter influenciado os resultados. Primeiro, no Experimento 1, os participantes se sentavam em um único banco, situado à frente de uma mesa. Sobre a mesa havia uma única bandeja com cinco utensílios, cada um contendo uma fruta específica, sendo esses utensílios partilhados por todos os participantes. No Experimento 2, os participantes se sentavam separadamente em carteiras, ainda que todos juntos na sessão, e cada participante tinha sua própria bandeja com os cinco utensílios contendo frutas diferentes. Foi observada uma maior interação entre os participantes do Experimento 1, o que pode ter contribuído para os resultados. Por exemplo, na Fase 5 - LBII desse experimento, houve consumo de frutas em uma única sessão por três participantes (AV, RA e RE). Nessa ocasião, os pesquisadores observaram que, após as instruções, as crianças passaram vários minutos sem consumir frutas, mas assim que uma delas comeu um pedaço, as outras duas fizeram o mesmo. Esses resultados são consistentes com a sugestão de Hendy e Raudenbush (2000) de que crianças em idade pré-escolar podem aprender preferências alimentares por meio da imitação de seus pares (ver também Lowe et al., 1998).

Segundo, aparentemente, não havia diferenças socioeconômicas entre os participantes da escola pública e privada, visto que ambas eram localizadas na mesma região urbana. No entanto, uma avaliação específica sobre a renda familiar de cada participante não foi realizada. Caso os participantes da escola pública e da escola privada tenham diferido no que se refere à renda familiar, é possível que os brinquedos utilizados não tenham apresentado o mesmo valor reforçador para esses dois grupos.

Terceiro, no presente estudo, a ordem de apresentação das intervenções não foi manipulada, de modo que a contribuição dessa variável não foi diretamente avaliada. Apesar disso, os resultados obtidos foram consistentes com aqueles observados com crianças de outros países.

Além disso, o consumo de frutas variou para um mesmo participante ao longo das diferentes sessões de cada condição experimental e entre participantes em uma mesma condição. A variabilidade intrassujeito observada é característica de comportamentos em transição, em que o controle pela contingência em vigor ainda não está fortemente estabelecido. Variáveis não avaliadas no presente estudo, como o histórico de consumo de frutas e o nível de privação no início de cada sessão, bem como a seleção dos participantes a partir de relatos das escolas podem ser fontes de variabilidade intrassujeito. Quanto à variabilidade entre sujeitos, é possível se inferir que, além das variáveis acima, a presença da experimentadora possa ter influenciado o consumo, conforme já apontado por Kroger-Costa e AbreuRodrigues (2009), ao demonstrarem efeitos da presença do experimentador sobre a sensibilidade do responder a alterações nas contingências.

Finalmente, em ambos os experimentos, na segunda linha de base, o aumento de consumo observado nas fases de intervenção anteriores não se manteve, embora o consumo nessa fase tenha sido maior do que na primeira linha de base. Esses resultados corroboram as argumentações feitas por Taylor e Upton (2015), em uma revisão sistemática do Food Dudes, a qual indicou que o programa é eficaz no curto prazo, mas que sua eficácia no longo prazo não foi ainda demonstrada nos follow-ups. O procedimento de mensurar o consumo em follow-ups de curto e longo prazo não foi realizado no presente estudo em decorrência do limite de tempo imposto pelas escolas para execução do mesmo. 
Segundo Horne et al. (2011), poucos estudos apresentam evidências de manutenção do consumo alimentar por seis meses após as intervenções. Um dos estudos com melhores resultados de manutenção após 12 meses de follow-up (Horne et al., 2004) envolvia, além da intervenção na escola, intervenção parental em ambiente doméstico, procedimento este também utilizado com alguns grupos no Programa Food Dudes. Sugere-se, para pesquisas futuras, a avaliação do efeito da inclusão de intervenções também em ambiente doméstico com participação parental.
Visto que o consumo de frutas é de interesse de várias instâncias sociais, por envolver aspectos relacionados à saúde das crianças, e que seu baixo consumo pode acarretar o desenvolvimento de doenças crônicas não transmissíveis (DCNT), estudos com essa temática podem contribuir para a compreensão da aprendizagem de consumo alimentar em crianças. O presente estudo aponta resultados favoráveis para estabelecer o comportamento de comer frutas por meio do uso de modelação e consequências, principalmente quando essas estratégias são combinadas.

\section{REFERÊNCIAS}

Baranowski, T. (2000). Physical activity and nutrition in children and youth: An overview of obesity prevention. Journal of Preventive Medicine, 31, 1-10. https://doi.org/10.1006/ pmed.2000.0686

Barrett, B. H. (1962). Reduction in rate of multiple tics by free operant conditioning methods. Journal of Nervous and Mental Disease, 135, 187-195. https://doi.org/10.1097/00005053196213530-00001

Bigio, R. S., Verly Jr., E., Castro, M. A, César, C. L. G., Fisberg, R. M., \& Marchioni, D. M. L. (2010). Determinantes do consumo de frutas e hortaliças em adolescentes por regressão quantílica. Saúde Pública, 45, 448-456.

Birch, L. L. (1987). The role of experience in children's food acceptance patterns. Journal of the American Dietetic Association, 87, S36-S40.

Birch, L. L., \& Marlin, D. W. (1982). I don't like it, I never tried it: effects of exposure on two-year-old children's food preferences. Appetite, 3, 353-360. https://doi.org/10.1016/ S0195-6663(82)80053-6

Borzekowski, D. L. G., \& Robinson, T. N. (2001). The 30-second effect: An experiment revealing the impact of television commercials on food preferences of preschoolers. Journal of the American Dietetic Association, 101, 42-46. https://doi. org/10.1016/S0002-8223(01)00012-8

Cameron, J., Banko, K. M., \& Peirce, W. D. (2001). Pervasive negative effects of rewards on intrinsic motivation: The myth continues. The Behavior Analyst, 24, 1-44. https://doi. org/10.1007/BF03392017

Catania, A. C. (1998). Learning. Prentice Hall.

Cooke, L. (2007). The importance of exposure for healthy eating in childhood: A review. Journal of Human Nutrition and Dietetics, 20, 294-301. https://doi.org/10.1111/j.1365277X.2007.00804.X

Cooper J. O., Heron, T. E., \& Heward, W. L. (2007). Applied Behavior Analysis (2 ${ }^{\mathrm{a}}$ ed.). Prentice Hall.

Costa, L. C. F. (2012). Fatores associados ao consumo adequado de frutas e hortaliças em escolares de Santa Catarina, Brasil. Cadernos de Saúde Pública, 28, 1133-1142.

Damiani, F. T., Pereira, P. L., \& Ferreira, G. M. (2017). Consumo de frutas, legumes e verduras na Região Centro-Oeste do Brasil: prevalência e fatores associados. Ciência \& Saúde Coletiva, 22, 369-382. https://doi.org/10.1590/141381232017222.12202015

Deci, E. L., Koestner, R., \& Ryan, R. M. (1999). A meta-analytic review of experiments examining the effects of extrinsic rewards on intrinsic motivation. Psychological Bulletin, 125, 627-668.

Dickinson, A. M. (1989). The detrimental effects of extrinsic reinforcement on 'intrinsic motivation'. The Behavior Analyst, 12, 1-15. https://doi.org/10.1007/BF03392473
Domel, S. B., Baranowski, T., Davies, H., Thompson, W. O., Leonard, S. B., Riley, P., et al. (1993). Development and evaluation of a school intervention to increase fruit and vegetables consumption among 4th and 5th grade students. Journal of Nutrition Education and Behavior, 25, 345-349. https://doi.org/10.1016/S0022-3182(12)80224-X

Foerster, S. B., Gregson, J., Beall, D. L., Hudes, M., Magnuson, H., Livingstone, S., et al. (1998). The California children's 5 a day-power play! campaign: Evaluation of a largescale social marketing initiative. Family and Community Health, 21, 46-64.

Erjavec, M., Viktor, S., Horne, P., \& Lowe, F. (2012). Given that many of our attitudes to health are set in childhood and that food consumption patterns are established early in life, it is clearly important that any attempts to produce long-term improvements in the nation's diet should start with children. Community Practitioner, 85, 40-42.

FAO - Food and Agriculture Organization (2007). Socio-economic analysis and policy implications of the roles of agriculture in developing countries. Summary Report, Roles of Agriculture Project. FAO

Gomes, F. S., Castro, I. R. R., \& Cruz, R. (2006). Promoción de frutas y hortalizas en Brasil: la contribución del programa 5 al día. Revista Chilena de Nutrición, 33, 95-299. http://dx.doi. org/10.4067/S0717-75182006000300013

Grubliauskiene, A., Verhoeven, M., \& Dewitte, S. (2012). The joint effect of tangible and non-tangible rewards on healthy food choices in children. Appetite, 59, 403-408. https://doi. org/10.1016/j.appet.2012.06.003

Halpern, G. (2003). Comerciais veiculados em programação infantojuvenil de canais abertos de TV e sua relação com a escolha de alimentos em amostra de escolares. [Dissertação de Mestrado, Universidade Federal de São Paulo] Repositório Institucional Unifesp. https://repositorio.unifesp.br/handle/11600/19752

Hendy, H. M., \& Raudenbush, B. (2000). Effectiveness of teacher modelling to encourage food acceptance in preschool children. Appetite, 34, 61-76. https://doi.org/10.1006/appe.1999.0286

Horne, P. J., \& Lowe, C. F. (1998). The way to healthy eating for children. British Food Journal, 100, 133-140. https://doi. org/10.1108/00070709810207496

Horne, P. J., Greenhalgh, J., Erjavec, M., Lowe, C. F., Viktor, S., \& Whittaker, C. J. (2011). Increasing pre-school children's consumption of fruit and vegetables. A modelling and rewards intervention. Appetite, 56, 375-385. https://doi.org/10.1016/j. appet.2010.11.146

Horne, P. J., Lowe, C. F., Fleming, P. F. J, \& Dowey, A. J. (1995). An effective procedure for changing food preferences in 5-7-yearold children. Proceedings of the Nutrition Society, 54, 441-452. https://doi.org/10.1079/PNS19950013

Horne, P. J., Tapper, K., Bowdery, M., \& Egerton, C. (1998). The way to healthy eating for children. British Food Journal, 100, 133-140. https://doi.org/10.1108/00070709810207496 
Horne, P., Tapper, K., Lowe, C. F., Hardman, C. A., Jackson, M. C., \& Woolner, J. (2004). Increasing children's fruit and vegetable consumption: A peer-modeling and rewards-based intervention. European Journal of Clinical Nutrition, 8, 1649-1660. https:// doi.org/10.1038/sj.ejen. 1601838

Horne, P. J., Hardman, C. A., Lowe, C. F., \& Rowlands, A. V. (2009). Increasing children's physical activity: A peer modelling, rewards and pedometer-based intervention. European Journal of Clinical Nutrition, 63, 191-198. https://doi.org/10.1038/ sj.ejen. 1602915

Johnston, J. M., \& Pennypacker, H. S. (1993). Readings for strategies and tactics of behavioral research $\left(2^{\mathrm{a}} \mathrm{ed}.\right)$. Erlbaum.

Kazdin, A. E. (1977). The token economy: a review and evaluation. Plenum.

Kroger-Costa, A., \& Abreu-Rodrigues, J. (2012). Effects of historical and social variables on instruction following. The Psychological Record, 62, 691-706. https://doi.org/10.1007/ BF03395829

Lakkakula, A., Geaghan, J. P., Wong, W., Zanovec, M., Pierce, S. H., \& Tuuri, G. (2011). A cafeteria-based tasting program increased liking of fruits and vegetables by lower, middle and upper elementary school-age children. Appetite, 57, 299-302. https://doi.org/10.1016/j.appet.2011.04.010

Lowe, C. F., Dowey, A., \& Horne, P. (1998). Changing what children eat. In A. Murcott (Ed.), The national's diet: The social science of food choice (pp. 57-80). Addison Wesley Longman.

Lowe, C. F., Horne, P. J., Tapper, K., Bowdery, M., \& Egerton, C. (2004). Effects of a peer modelling and rewards-based intervention to increase fruit and vegetable consumption in children. European Journal of Clinical Nutrition, 58, 510-522. https://doi.org/10.1016/j.appet.2011.04.010

Mikkilä, V., Räsänen, L., Raitakari, O. T., Pietinen, P., \& Vikari, J. (2004). Longitudinal changes in diet from childhood into adulthood with respect to risk of cardiovascular diseases: The cardiovascular risk in young finns study. European Journal of Clinical Nutrition, 58, 1038-1045. https://doi.org/10.1038/ sj.ejen.1601929

Ministério da Saúde (2005). Guia alimentar para a população brasileira: promovendo a alimentação saudável. Secretaria de Atenção à Saúde, Coordenação-Geral da Política de Alimentação e Nutrição.

Ministério da Saúde. (2009). Consumo de frutas e hortaliças é menor que o recomendado pela OMS. http://bvsms.saude.gov. $\mathrm{br} / \mathrm{bvs} / \mathrm{sus} / \mathrm{pdf} / \mathrm{setembro/consumo} \mathrm{frutas}$ hortalicas menor recomendado_oms_2209.pdf

Ministério da Saúde (2014). Vigitel - Vigilância de fatores de risco e proteção para doenças crônicas por inquérito telefônico. Recuperado de http://portalsaude.saude.gov.br/ images/ pdf/2014/abril/30/Lancamento-Vigitel-28-04-ok.pdf.

Moura, N. M. (2010). Influência da mídia no comportamento alimentar de crianças e adolescentes. Segurança Alimentar e Nutricional, 17, 113-122. https://doi.org/10.20396/san. v17i1.8634805

Pereira, S. T., Pereira, C. R., \& Angelis-Pereira, C. M. (2017). Influência de intervenções educativas no conhecimento sobre alimentação e nutrição de adolescentes de uma escola pública. Ciência \& Saúde Coletiva, 22, 427-435. https://doi. org/10.1590/1413-81232017222.16582015

Presti, G., Cau, S., Oppo, A., \& Moderato, P. (2015). Increased classroom consumption of home-provided fruits and vegetables for normal and overweight children: Results of the Food Dudes
Program in Italy. Journal of Nutrition Education and Behavior, 47, 338-344. https://doi.org/10.1016/j.jneb.2015.04.331

Prevedini, B. A., \& Carnevali, D. (2016). Dalla teoria del comportamento all'analisi applicata: quattro modelli in diversi ambiti applicativi. Acta Comportamentalia, 24, 169-193.

Rosales-Ruiz, J., \& Baer, D. M. (1997). Behavioral cusps: A developmental and pragmatic concept for behavior analysis. Journal of Applied Behavior Analysis, 30, 533-544. https://doi. org/10.1901/jaba.1997.30-533

Saraiva, A. D., Cabral, C. B., Moraes, C. C. H., Nascimento., S. L., Silva., C. M. L., Frazão, F. G. A. et al. (2015). Características antropométricas e hábitos alimentares de escolares. Ciência \& Saúde, 8, 59-66. https://doi.org/10.15448/1983652X.2015.2.19657

SBP - Sociedade Brasileira de Pediatria (2012). Pirâmide alimentar. http://www.sbp.com.br/fileadmin/user_upload/pdfs/14297ecartazPiramide.pdf.

Singer, M. R., Moore, L. L., Garahie, E. J., \& Ellison, R. C. (1995). The tracking of nutrient intake in young children: The Framingham Children's Study. American Journal of Public Health, 85, 1673-1677. https://doi.org/10.2105/ AJPH.85.12.1673

Skinner, B. F. (1980). Contingências do reforço: uma análise teórica (Coleção Os Pensadores). Abril Cultural.

Staiano, E. A., Marker, M. A., Frelier, M. J., Hsia, S. D., \& Martin, K. C. (2016). Influence of screen-based peer modeling on preschool children's vegetable consumption and preferences. Journal of Nutrition Education and Behavior, 48(5), 331-335. https://doi.org/10.1016/j.jneb.2016.02.005

Stice, E. (1998). Relation of media exposure to eating disorder symptomatology: An examination of mediating mechanisms. Journal of Abnormal Psychology, 103, 31-42. https://doi. org/10.1037/0021-843X.103.4.836

Taylor, C., \& Upton, P. (2015). Increasing primary school children's fruit and vegetable consumption. A review of the food dudes program. Health Education, 115, 178-196. https://doi. org/10.1108/HE-02-2014-0005

Ueda, M. H., Porto, R. B., \& Vasconcelos, L. A. (2014). Publicidade de alimentos e escolhas alimentares de crianças. Psicologia: Teoria e Pesquisa, 30, 53-61. https://doi.org/10.1590/S010237722014000100007

Upton, P., Taylor, C., \& Upton, D. (2015). The effects of the Food Dudes Programme on children's intake of unhealthy foods at lunchtime. Perspectives in Public Health, 135, 152-159. https:// doi.org/10.1177/1757913914526163

Wardle, J., Cooke, L. J., Gibson, E. L., Sapochnik, M., Sheiham, A., \& Lawson, M. (2003). Increasing children's acceptance of vegetables: A randomized trial of parent-led exposure. Appetite, 40, 155-162. https://doi.org/10.1016/S0195-6663(02)00135-6

Wengreen, J. H., Madden, J. G., Aguilar, S. S., Smits, R. R., \& Jones, A. B. (2013). Incentivizing children's fruit and vegetable consumption: Results of a United States pilot study of the Food Dudes Program. Journal of Nutrition Education and Behavior, 45, 54-59. https://doi.org/10.1016/j.jneb.2012.06.001

WHO - World Health Organization (2003). Diet, nutrition and the prevention of chronic diseases. Report of a Joint WHO/FAO Expert Consultation. Geneva.

WHO - World Health Organization (2014). Global status report on non-communicable diseases. http://apps.who.int/iris/ bitstream/10665/148114/1/9789241564854_eng.pdf. 